Document downloaded from:

http://hdl.handle.net/10251/55393

This paper must be cited as:

Andreu Estellés, C.; Cambil Teba, N.; Cordero Barbero, A.; Torregrosa Sánchez, JR. (2014). A class of optimal eighth-order derivative-free methods for solving the Danchick-Gauss problem. Applied Mathematics and Computation. 232:237-246. doi:10.1016/j.amc.2014.01.056.

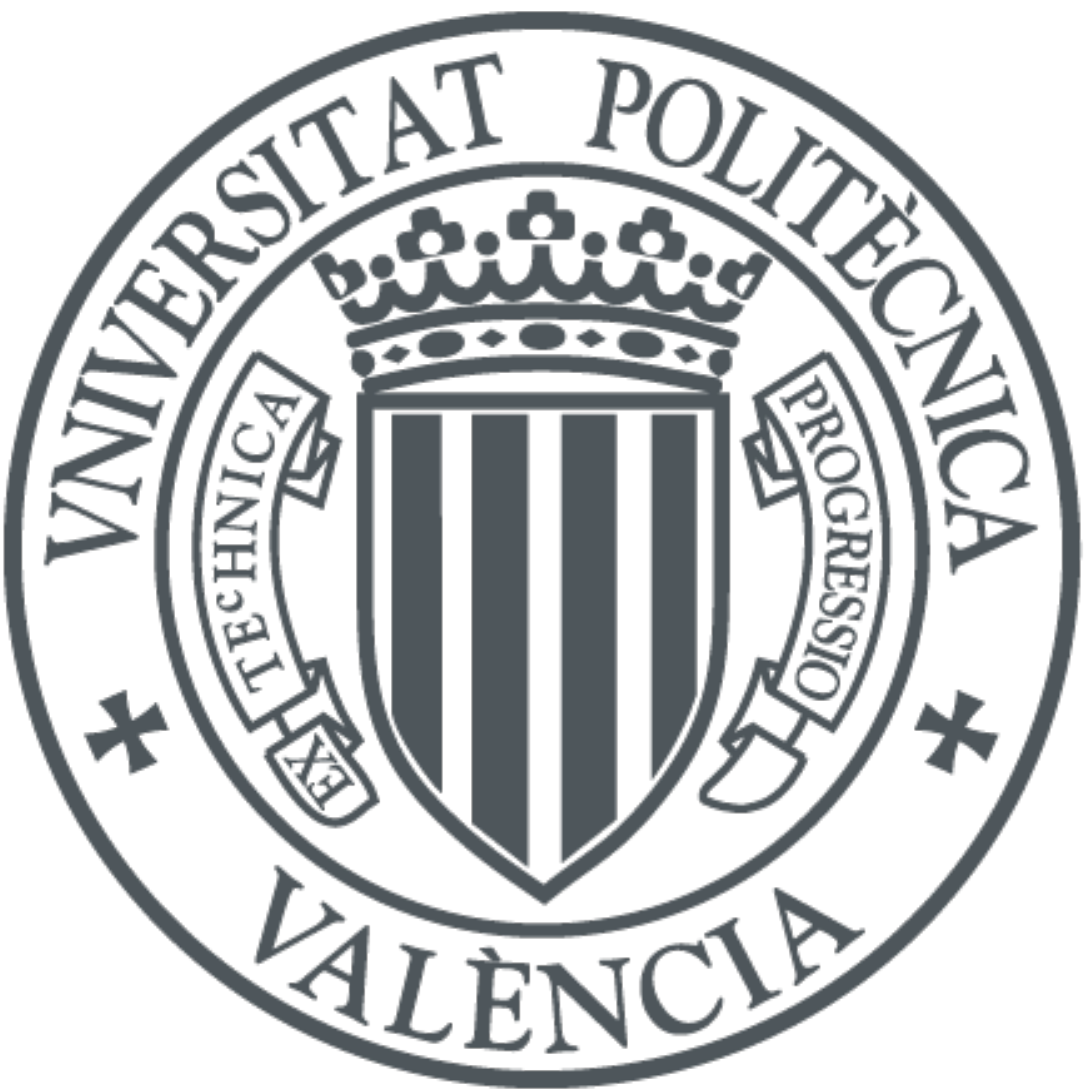

The final publication is available at

http://dx.doi.org/10.1016/j.amc.2014.01.056

Copyright Elsevier

Additional Information 


\title{
A class of optimal eighth-order derivative-free methods for solving the Danchick-Gauss problem 추
}

\author{
Carlos Andreu ${ }^{\mathrm{a}}$, Noelia Cambil ${ }^{\mathrm{a}}$, Alicia Cordero ${ }^{\mathrm{a}}$, Juan R. Torregrosa ${ }^{\mathrm{a}, *}$ \\ ${ }^{a}$ Instituto de Matemática Multidisciplinar \\ Universitat Politècnica de València \\ Camino de Vera s/n, 46022 València, Spain
}

\begin{abstract}
A derivative-free optimal eighth-order family of iterative methods for solving nonlinear equations is constructed using weight functions approach with divided first order differences. Its performance, along with several other derivativefree methods, is studied on the specific problem of Danchick's reformulation of Gauss' method of preliminary orbit determination. Numerical experiments show that such derivative-free, high-order methods offer significant advantages over both, the classical and Danchick's Newton approach.
\end{abstract}

Keywords: Nonlinear equation, iterative method, derivative-free scheme, order of convergence, basin of attraction, efficiency index

\section{Introduction}

Many applied problems in different fields of science and technology require to find the solution of a nonlinear equation $f(x)=0$, where $f: I \subset \mathbb{R} \rightarrow \mathbb{R}$ is a scalar function on an open interval $I$ : in particular, the numerical solution of nonlinear equations and systems are needed in the study of dynamical models of chemical reactors [1], or in radioactive transfer [2]. Moreover, many of numerical applications use high precision in their computations; in [3], high-precision calculations are used to solve interpolation problems in Astronomy; in [4] the authors describe the use of arbitrary precision computations to improve the results obtained in climate simulations; the results of these numerical experiments show that the high order methods associated with a multiprecision arithmetic floating point are very useful, because it yields a clear reduction in iterations.

The performance of an iterative method can be measured by the efficiency index introduced by Ostrowski in [5]. In this sense, Kung and Traub conjectured in [6] that a multipoint method without memory performing $n+1$ functional evaluations per iteration has at most convergence order $2^{n}$. A method is called optimal when its order reaches this bound. In [7] we can see an unified presentation of many multipoint iterative schemes designed during the last years.

Since Steffensen designed (see, for example [8]) his second-order method as a variant of Newton's scheme without derivatives, many optimal fourth-order methods have been proposed in order to improve the convergence properties of it (see, for example $[9,10]$ and the references therein). More limited is the quantity of optimal eighth-order derivative-free iterative methods, mainly designed by using divided differences of high order (see, for example, [10, 11, 12]). Moreover, when only first-order divided differences are used, it is necessary to introduce several weight functions with one, two or more variables, all of them quotients of function $f$ evaluated in different steps of the process (see, for instance $[13,14,15]$ and the references therein).

By using weight functions procedure, with functions of one variable, and divided differences of order one, we derive an Steffensen-type family of optimal eighth-order schemes. We would like to remark that we use a quotient of divided differences as a variable of one of the weight function used in our process. In the numerical section we use some special

\footnotetext{
This research was supported by Ministerio de Ciencia y Tecnología MTM2011-28636-C02-02

* Corresponding author

Email addresses: caranesandreu@gmail.com (Carlos Andreu), nctnoelia@gmail.com (Noelia Cambil), acordero@mat.upv. es (Alicia Cordero), jrtorre@mat. upv. es (Juan R. Torregrosa )
} 
cases of this family for solving the classical problem of preliminary orbit determination of artificial satellites, improving the classical Gauss's method and the iterative scheme designed by Danchick in [16], that removed several limitations of the original methods.

The rest of the paper is organized as follow. In Section 2 we describe our family of iterative methods and prove that, under some conditions of the weight functions, all of them have order of convergence eight, needing four functional evaluations per iteration, that is, they are optimal schemes in the sense of Kung-Traub's conjecture. Numerical results given in Section 3 allow to compare the proposed methods with other ones, into the Danchick's algorithm, for solving the problem of the determination of artificial satellites. We also analyze the basins of attraction of the different methods used in this work. Both studies confirm the theoretical results and show the high stability of the proposed schemes. We finish the paper with some conclusions and the references used in it.

\section{A new family of Steffensen-type methods with optimal order of convergence.}

We design a three-step family of Steffensen-type methods, using weight functions technique. To develop it, we compose Steffensen's method with itself three times, but using different divided differences in each step. For reaching the optimal order, we use in the second step a weight function whose variable is a quotient of two values of $f$ and in the third step a weight function whose variable is the quotient between two divided difference. The iterative expression of our family is:

$$
\begin{aligned}
y_{k} & =x_{k}-\frac{f\left(x_{k}\right)}{f\left[z_{k}, x_{k}\right]}, \\
w_{k} & =y_{k}-H(\mu) \frac{f\left(y_{k}\right)}{f\left[y_{k}, z_{k}\right]}, \quad \mu=\frac{f\left(y_{k}\right)}{f\left(z_{k}\right)} \\
x_{k+1} & =w_{k}-G(\eta) \frac{f\left(w_{k}\right)}{f\left[w_{k}, y_{k}\right]}, \quad \eta=\frac{f\left[w_{k}, y_{k}\right]}{f\left[w_{k}, z_{k}\right]}
\end{aligned}
$$

where $z_{k}=x_{k}+f\left(x_{k}\right)^{3}, f[\cdot, \cdot]$ denotes divided difference of first order and $H(\mu)$ and $G(\eta)$ are real functions.

In this scheme, the election of the exponent of $f\left(x_{k}\right)$ in the expression of $z_{k}$ is key to obtain the order of convergence eight. In fact, following the ideas shown in [17], if we take in (1) $z_{k}=x_{k}+f\left(x_{k}\right)$ or $z_{k}=x_{k}+f\left(x_{k}\right)^{2}$ the order eight is not reached. On the other hand, the next result holds also if $z_{k}=x_{k}+\gamma f\left(x_{k}\right)^{n}$, for $n \geq 3$ and any value of parameter $\gamma, \gamma \neq 0$.

Theorem 1. Let $x^{*} \in I$ be a simple zero of a sufficiently differentiable function $f: I \subseteq \mathbb{R} \rightarrow \mathbb{R}$ in a open interval I. Let us consider $x_{0}$ close enough to $x^{*}$ and $H$ and $G$ sufficiently differentiable real functions satisfying the following conditions: $H(0)=0, H^{\prime}(0)=1, G(1)=1, G^{\prime}(1)=0, G^{\prime \prime}(1)=2$ and $G^{\prime \prime \prime}(1)=-12$. The iterative methods (1) have optimal eighth-order of convergence, whose error equation is:

$$
e_{k+1}=\frac{1}{2} C_{2}\left(\left(-6+H_{2}\right) C_{2}^{2}+2 C_{3}\right)\left(f^{\prime}\left(x^{*}\right)^{3} C_{2}^{2}-4 C_{2}^{4}+C_{3}^{2}-C_{2} C_{4}\right) e_{k}^{8}+O\left[e_{k}^{9}\right],
$$

where $e_{k}=x_{k}-x^{*}$ and $C_{k}=\frac{1}{k !} \frac{f^{(k)}\left(x^{*}\right)}{f^{\prime}\left(x^{*}\right)}, k=2,3, \ldots$

Proof: Expanding $f\left(x_{k}\right)$ in Taylor's series around $x=x^{*}$ and taking into account that $e_{k}=x_{k}-x^{*}$ and $f\left(x^{*}\right)=0$,

$$
f\left(x_{k}\right)=f^{\prime}\left(x^{*}\right)\left[e_{k}+C_{2} e_{k}^{2}+C_{3} e_{k}^{3}+C_{4} e_{k}^{4}+C_{5} e_{k}^{5}\right]+O\left[e_{k}^{6}\right]
$$

and

$$
z_{k}-x^{*}=e_{k}+f^{\prime}\left(x^{*}\right)^{3}\left[e_{k}^{3}+3 C_{2} e_{k}^{4}+\left(3 C_{2}^{2}+3 C_{3}\right) e_{k}^{5}\right]+O\left[e_{k}^{6}\right]
$$


Therefore,

$$
\begin{aligned}
f\left(z_{k}\right) & =f^{\prime}\left(x^{*}\right)\left[e_{k}+C_{2} e_{k}^{2}+\left(f^{\prime}\left(x^{*}\right)^{3}+C_{3}\right) e_{k}^{3}+\left(5 f^{\prime}\left(x^{*}\right)^{3} C_{2}+C_{4}\right) e_{k}^{4}\right. \\
& \left.+\left(9 f^{\prime}\left(x^{*}\right)^{3} C_{2}^{2}+6 f^{\prime}\left(x^{*}\right)^{3} C_{3}+C_{5}\right) e_{k}^{5}\right]+O\left[e_{k}^{6}\right] .
\end{aligned}
$$

Now, we get the Taylor's expansion of $f\left[z_{k}, x_{k}\right]=\frac{f\left(z_{k}\right)-f\left(x_{k}\right)}{z_{k}-x_{k}}$, by replacing in it the equations (2), (3) and (4).

$$
\begin{aligned}
f\left[z_{k}, x_{k}\right] & =f^{\prime}\left(x^{*}\right)+2 f^{\prime}\left(x^{*}\right) C_{2} e_{k}+3 f^{\prime}\left(x^{*}\right) C_{3} e_{k}^{2}+\left(f^{\prime}\left(x^{*}\right)^{4} C_{2}+4 f^{\prime}\left(x^{*}\right) C_{4}\right) e_{k}^{3} \\
& +\left(3 f^{\prime}\left(x^{*}\right)^{4} C_{2}^{2}+3 f^{\prime}\left(x^{*}\right)^{4} C_{3}+5 f^{\prime}\left(x^{*}\right) C_{5}\right) e_{k}^{4} \\
& +3 f^{\prime}\left(x^{*}\right)\left(f^{\prime}\left(x^{*}\right)^{3} C_{2}^{3}+4 f^{\prime}\left(x^{*}\right)^{3} C_{2} C_{3}+2\left(f^{\prime}\left(x^{*}\right)^{3} C_{4}+C_{6}\right)\right) e_{k}^{5}+O\left[e_{k}^{6}\right] .
\end{aligned}
$$

Then, the second order of convergence of first step of our iterative process (1) is obtained

$$
\begin{aligned}
y_{k}-x^{*} & =C_{2} e_{k}^{2}+\left(-2 C_{2}^{2}+2 C_{3}\right) e_{k}^{3}+\left(4 C_{2}^{3}+C_{2}\left(f^{\prime}\left(x^{*}\right)^{3}-7 C_{3}\right)+3 C_{4}\right) e_{k}^{4} \\
& +\left(-8 C_{2}^{4}+3 f^{\prime}\left(x^{*}\right)^{3} C_{3}+20 C_{2}^{2} C_{3}-6 C_{3}^{2}-10 C_{2} C_{4}+4 C_{5}\right) e_{k}^{5}+O\left[e_{k}^{6}\right] .
\end{aligned}
$$

So,

$$
\begin{aligned}
f\left(y_{k}\right) & =f^{\prime}\left(x^{*}\right)\left[C_{2} e_{k}^{2}-2\left(\left(C_{2}^{2}-C_{3}\right)\right) e_{k}^{3}+\left(5 C_{2}^{3}+C_{2}\left(f^{\prime}\left(x^{*}\right)^{3}-7 C_{3}\right)+3 C_{4}\right) e_{k}^{4}\right. \\
& \left.+\left(-12 C_{2}^{4}+3 f^{\prime}\left(x^{*}\right)^{3} C_{3}+24 C_{2}^{2} C_{3}-6 C_{3}^{2}-10 C_{2} C_{4}+4 C_{5}\right) e_{k}^{5}\right]+O\left[e_{k}^{6}\right] .
\end{aligned}
$$

Now, we can express the variable $\mu=\frac{f\left(y_{k}\right)}{f\left(z_{k}\right)}$, in terms of the error $e_{k}$, as

$$
\begin{aligned}
\mu & =C_{2} e_{k}+\left(-3 C_{2}^{2}+2 C_{3}\right) e_{k}^{2}+\left(8 C_{2}^{3}-10 C_{2} C_{3}+3 C_{4}\right) e_{k}^{3} \\
& +\left(-20 C_{2}^{4}+f^{\prime}\left(x^{*}\right)^{3} C_{3}-8 C_{3}^{2}+C_{2}^{2}\left(-2 f^{\prime}\left(x^{*}\right)^{3}+37 C_{3}\right)-14 C_{2} C_{4}+4 C_{5}\right) e_{k}^{4} \\
& +\left(48 C_{2}^{5}+2 C_{2}^{3}\left(2 f^{\prime}\left(x^{*}\right)^{3}-59 C_{3}\right)+51 C_{2}^{2} C_{4}+\left(3 f^{\prime}\left(x^{*}\right)^{3}-22 C_{3}\right) C_{4}\right. \\
& \left.+C_{2}\left(-9 f^{\prime}\left(x^{*}\right)^{3} C_{3}+55 C_{3}^{2}-18 C_{5}\right)+5 C_{6}\right) e_{k}^{5}+O\left[e_{k}^{6}\right] .
\end{aligned}
$$

Since $\mu \longrightarrow 0$ when $k \longrightarrow \infty$, we expand the weight function $H(\mu)$ in Taylor's series around zero.

$$
\begin{aligned}
H(\mu) & \left.=H_{0}+H_{1} C_{2} e_{k}+\frac{1}{2}\left(-6 H_{1}+H_{2}\right) C_{2}^{2}+2 H_{1} C_{3}\right) e_{k}^{2} \\
& +\left(\left(8 H_{1}-3 H_{2}+\frac{H_{3}}{6}\right) C_{2}^{3}+2\left(-5 H_{1}+H_{2}\right) C_{2} C_{3}+3 H_{1} C_{4}\right) e_{k}^{3} \\
& +\left(-\frac{1}{2}\left(40 H_{1}-25 H_{2}+3 H_{3}\right) C_{2}^{4}+f^{\prime}\left(x^{*}\right)^{3} H_{1} C_{3}+\left(-8 H_{1}+2 H_{2}\right) C_{3}^{2}\right. \\
& \left.+C_{2}^{2}\left(-2 f^{\prime}\left(x^{*}\right)^{3} H_{1}+\left(37 H_{1}-16 H_{2}+H_{3}\right) C_{3}\right)+\left(-14 H_{1}+3 H_{2}\right) C_{2} C_{4}+4 H_{1} C_{5}\right) e_{k}^{4}+O\left[e_{k}^{5}\right],
\end{aligned}
$$

where $H_{0}=H(0), H_{1}=H^{\prime}(0)$ and $H_{2}=H^{\prime \prime}(0)$. So, the error expression of the second step of (1) is:

$$
\begin{aligned}
w_{k}-x^{*} & =-\left(-1+H_{0}\right) C_{2} e_{k}^{2}+\left(\left(-2+3 H_{0}-H_{1}\right) C_{2}^{2}-2\left(-1+H_{0}\right) C_{3}\right) e_{k}^{3} \\
& +\left(\left(4-7 H_{0}+6 H_{1}-\frac{H_{2}}{2}\right) C_{2}^{3}+C_{2}\left(-f^{\prime}\left(x^{*}\right)^{3}\left(-1+H_{0}\right)\right.\right. \\
& \left.\left.+\left(-7+10 H_{0}-4 H_{1}\right) C_{3}\right)-3\left(-1+H_{0}\right) C_{4}\right) e_{k}^{4}+O\left[e_{k}^{5}\right] .
\end{aligned}
$$

In order to achieve optimal fourth-order of convergence in the second step, we require $H_{0}=1$ and $H_{1}=1$. Then,

$$
w_{k}-x^{*}=\left(-\frac{1}{2}\left(-6+H_{2}\right) C_{2}^{3}-C_{2} C_{3}\right) e_{k}^{4}+O\left[e_{k}^{5}\right]
$$


By using the Taylor's expansion of $f\left(w_{k}\right), f\left[w_{k}, y_{k}\right]$ and $f\left[w_{k}, z_{k}\right]$ around $x^{*}$,

$$
\begin{gathered}
f\left(w_{k}\right)=-\frac{1}{2}\left(f^{\prime}\left(x^{*}\right)\left(\left(-6+H_{2}\right) C_{2}^{3}+2 C_{2} C_{3}\right)\right) e_{k}^{4} \\
+f^{\prime}\left(x^{*}\right)\left(\left(-18+\frac{9 H_{2}}{2}-\frac{H_{3}}{6}\right) C_{2}^{4}-2 C_{3}^{2}\right. \\
\left.+C_{2}^{2}\left(f^{\prime}\left(x^{*}\right)^{3}+\left(20-3 H_{2}\right) C_{3}\right)-2 C_{2} C_{4}\right) e_{k}^{5}+O\left[e_{k}^{6}\right], \\
f\left[w_{k}, y_{k}\right]=f^{\prime}\left(x^{*}\right)+f^{\prime}\left(x^{*}\right) C_{2}^{2} e_{k}^{2}+2 f^{\prime}\left(x^{*}\right) C_{2}\left(-C_{2}^{2}+C_{3}\right) e_{k}^{3} \\
+\frac{1}{2} f^{\prime}\left(x^{*}\right) C_{2}\left(-\left(-14+H_{2}\right) C_{2}^{3}+2 C_{2}\left(f^{\prime}\left(x^{*}\right)^{3}-7 C_{3}\right)+6 C_{4}\right) e_{k}^{4} \\
+\frac{1}{6} f^{\prime}\left(x^{*}\right) C_{2}\left(\left(-156+27 H_{2}-H_{3}\right) C_{2}^{4}+6 C_{2}^{2}\left(f^{\prime}\left(x^{*}\right)^{3}-3\left(-12+H_{2}\right) C_{3}\right)\right. \\
\left.-72 C_{2} C_{4}+6\left(3 f^{\prime}\left(x^{*}\right)^{3} C_{3}-4 C_{3}^{2}+4 C_{5}\right)\right) e_{k}^{5}+O\left[e_{k}^{6}\right]
\end{gathered}
$$

and

$$
\begin{aligned}
f\left[w_{k}, z_{k}\right] & =f^{\prime}\left(x^{*}\right)+f^{\prime}\left(x^{*}\right) C_{2} e_{k}+f^{\prime}\left(x^{*}\right) C_{3} e_{k}^{2}+f^{\prime}\left(x^{*}\right)\left(f^{\prime}\left(x^{*}\right)^{3} C_{2}+C_{4}\right) e_{k}^{3} \\
& +\frac{1}{2} f^{\prime}\left(x^{*}\right)\left(-\left(-6+H_{2}\right) C_{2}^{4}+C_{2}^{2}\left(6 f^{\prime}\left(x^{*}\right)^{3}-2 C_{3}\right)+2\left(2 f^{\prime}\left(x^{*}\right)^{3} C_{3}+C_{5}\right)\right) e_{k}^{4} \\
& +\frac{1}{6} f^{\prime}\left(x^{*}\right)\left(\left(-108+27 H_{2}-H_{3}\right) C_{2}^{5}+18 C_{2}\left(3 f^{\prime}\left(x^{*}\right)^{3}-C_{3}\right) C_{3}\right. \\
& +3 C_{2}^{3}\left(8 f^{\prime}\left(x^{*}\right)^{3}+\left(46-7 H_{2}\right) C_{3}\right)-12 C_{2}^{2} C_{4}+6\left(3 f^{\prime}\left(x^{*}\right)^{3} C_{4}+C_{6}\right) e_{k}^{5}+O\left[e_{k}^{6}\right],
\end{aligned}
$$

we get

$$
\begin{aligned}
\eta=\frac{f\left[w_{k}, y_{k}\right]}{f\left[w_{k}, z_{k}\right]} & =1-C_{2} e_{k}+\left(2 C_{2}^{2}-C_{3}\right) e_{k}^{2}+\left(-4 C_{2}^{3}-C_{2}\left(f^{\prime}\left(x^{*}\right)^{3}-4 C_{3}\right)-C_{4}\right) e_{k}^{3} \\
& +\left(8 C_{2}^{4}-2 f^{\prime}\left(x^{*}\right)^{3} C_{3}-12 C_{2}^{2} C_{3}+C_{3}^{2}+5 C_{2} C_{4}-C_{5}\right) e_{k}^{4} \\
& +\left(-\frac{1}{2}\left(26+H_{2}\right) C_{2}^{5}-\frac{1}{2} C_{2}^{3}\left(4 f^{\prime}\left(x^{*}\right)^{3}-\left(56+H_{2}\right) C_{3}\right)-17 C_{2}^{2} C_{4}\right. \\
& \left.+\left(-3 f^{\prime}\left(x^{*}\right)^{3}+2 C_{3}\right) C_{4}-6 C_{2}\left(C_{3}^{2}-C_{5}\right)-C_{6}\right) e_{k}^{5}+O\left[e_{k}^{6}\right] .
\end{aligned}
$$

In the same way, we are going to obtain the Taylor's expansion of $G(\eta)$ around 1 , since in this case $\eta \longrightarrow 1$ when $k \longrightarrow \infty$. Denoting by $G_{0}=G(1), G_{1}=G^{\prime}(1), G_{2}=G^{\prime \prime}(1)$ and $G_{3}=G^{\prime \prime \prime}(1)$, we have

$$
\begin{aligned}
G(\eta) & =G_{0}-G_{1} C_{2} e_{k}+\left(\frac{1}{2}\left(4 G_{1}+G_{2}\right) C_{2}^{2}-G_{1} C_{3}\right) e_{k}^{2}+\left(-\frac{1}{6}\left(24 G_{1}\right.\right. \\
& \left.\left.+12 G_{2}+G_{3}\right) C_{2}^{3}+C_{2}\left(-f^{\prime}\left(x^{*}\right)^{3} G_{1}+\left(4 G_{1}+G_{2}\right) C_{3}\right)-G_{1} C_{4}\right) e_{k}^{3} \\
& +\left(\left(8 G_{1}+6 G_{2}+G_{3}\right) C_{2}^{4}-2 f^{\prime}\left(x^{*}\right)^{3} G_{1} C_{3}+\left(G_{1}+\frac{G_{2}}{2}\right) C_{3}^{2}\right. \\
& \left.+\frac{1}{2} C_{2}^{2}\left(2 f^{\prime}\left(x^{*}\right)^{3} G_{2}-\left(24 G_{1}+12 G_{2}+G_{3}\right) C_{3}\right)+\left(5 G_{1}+G_{2}\right) C_{2} C_{4}-G_{1} C_{5}\right) e_{k}^{4} \\
& +\frac{1}{2}\left(-\left(8\left(4 G_{2}+G_{3}\right)+G_{1}\left(26+H_{2}\right)\right) C_{2}^{5}+C_{2}^{3}\left(-f^{\prime}\left(x^{*}\right)^{3}\left(4 G_{1}+4 G_{2}+G_{3}\right)\right.\right. \\
& \left.+\left(8\left(6 G_{2}+G_{3}\right)+G_{1}\left(56+H_{2}\right)\right) C_{3}\right)-\left(34 G_{1}+14 G_{2}+G_{3}\right) C_{2}^{2} C_{4} \\
& +\left(-6 f^{\prime}\left(x^{*}\right)^{3} G_{1}+2\left(2 G_{1}+G_{2}\right) C_{3}\right) C_{4}+C_{2}\left(6 f^{\prime}\left(x^{*}\right)^{3} G_{2} C_{3}\right. \\
& \left.\left.-\left(12 G_{1}+10 G_{2}+G_{3}\right) C_{3}^{2}+2\left(6 G_{1}+G_{2}\right) C_{5}\right)-2 G_{1} C_{6}\right) e_{k}^{5}+O\left[e_{k}^{6}\right] .
\end{aligned}
$$


Finally, replacing (5), (6) and (7) in the last step of (1), we obtain:

$$
\begin{aligned}
e_{k+1} & =\frac{1}{2}\left(-1+G_{0}\right) C_{2}\left(\left(-6+H_{2}\right) C_{2}^{2}+2 C_{3}\right) e_{k}^{4} \\
& +\left(-\frac{1}{6}\left(108+3 G_{1}\left(-6+H_{2}\right)-27 H_{2}+G_{0}\left(-108+27 H_{2}-H_{3}\right)+H_{3}\right) C_{2}^{4}\right. \\
& +2\left(-1+G_{0}\right) C_{3}^{2}+C_{2}^{2}\left(-f^{\prime}\left(x^{*}\right)^{3}\left(-1+G_{0}\right)-\left(-20+G_{1}+G_{0}\left(20-3 H_{2}\right)\right.\right. \\
& \left.\left.\left.+3 H_{2}\right) C_{3}\right)+2\left(-1+G_{0}\right) C_{2} C_{4}\right) e_{k}^{5}+O\left[e_{k}^{6}\right] .
\end{aligned}
$$

If we set $G_{0}=1, G_{1}=0, G_{2}=2$ and $G_{3}=-12$ the error equation is:

$$
e_{k+1}=\frac{1}{2} C_{2}\left(\left(-6+H_{2}\right) C_{2}^{2}+2 C_{3}\right)\left(f^{\prime}\left(x^{*}\right)^{3} C_{2}^{2}-4 C_{2}^{4}+C_{3}^{2}-C_{2} C_{4}\right) e_{k}^{8}+O\left[e_{k}^{9}\right]
$$

which proves the order of convergence eight of this family.

We would like to emphasize that, this new family of Steffensen-type methods have four functional evaluations per iteration, so it is optimal in the sense of Kung-Traub's conjecture.

\section{Numerical results.}

The classical method for preliminary orbit determination was obtained by Carl F. Gauss in 1801 for deducing the orbit of the minor planet Ceres. This method starts from two position vectors, denoted by $\vec{r}_{1}$ and $\vec{r}_{2}$, of the satellite in its orbit and the time instants in which they were obtained. Then, the procedure is based on the rate $y$ between the triangle and the ellipse sector defined by the two position vectors (see Figure 1a). The complete procedure to obtain Gauss' equations can be found in [18].

The preliminary orbit is determined by considering only the gravitational forces of two bodies. This yields to a planar motion, described by a conic. Let us set a two-dimensional coordinate system (see Figure $1 \mathrm{~b}$ ), where the $\mathrm{X}$ axis points to the perigee of the orbit, the closest point of the conical orbit to the focus and center of the system, the Earth. In this figure the true anomaly $\nu$ and the eccentric anomaly $E$ can be observed. In order to place this orbit in the celestial sphere and determine completely the position of a body in the orbit, some elements (called orbital or keplerian elements) must be determined. These orbital elements are:

- $\Omega$, (right ascension of the ascending node): defined as the equatorial angle between the Vernal point $\gamma$ and the ascending node $N$; it orients the orbit in the equatorial plane.

- $\omega$, (argument of the perigee): defined as the angle of the orbital plane, centered at the focus, between the ascending node $N$ and the perigee of the orbit; it orients the orbit in its plane.

- i, (inclination): dihedral angle between the equatorial and the orbital planes.

- a, (semi-major axis): which sets the size of the orbit.

- e, (eccentricity): which gives the shape of the ellipse.

- $T_{0}$, (perigee epoch): time for the passing of the object over the perigee, to determine a reference origin in time. It can be denoted by a exact date, in Julian Days, or by the amount of time ago the object was over the perigee.

The equations that we have used are known as the First and Second Gauss' equations:

$$
y^{2}=\frac{m}{l+x}, \quad y^{2}(y-1)=m X
$$

Also, by combining them, the unified equation of Gauss is obtained as:

$$
y=1+X(l+x),
$$




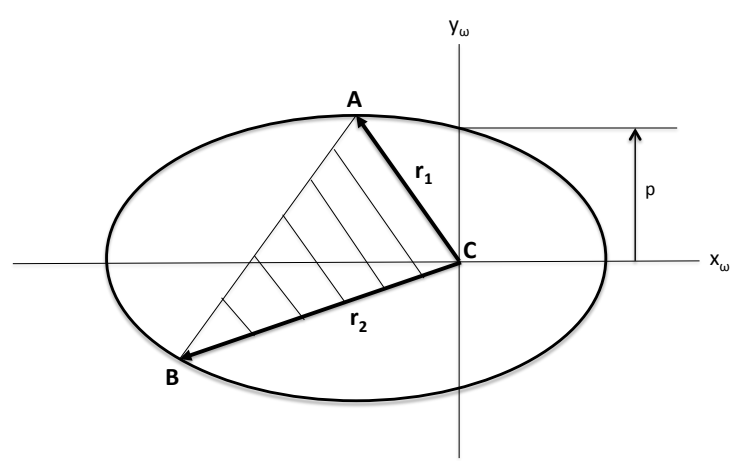

(a) Ratio sector-triangle $y$

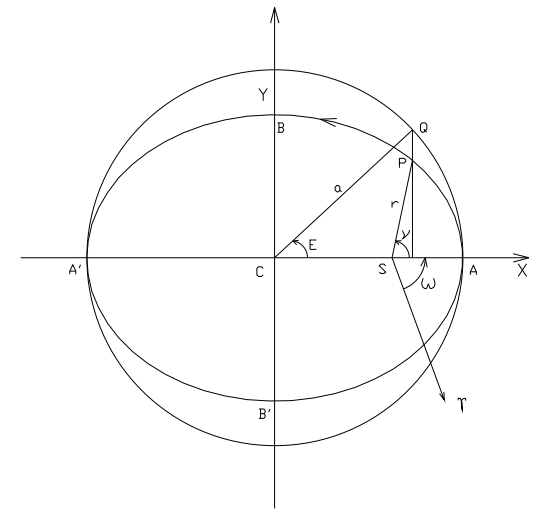

(b) Size, shape and anomalies

Figure 1: Some aspects of the orbital plane

where

$$
\begin{aligned}
& l=\frac{r_{1}+r_{2}}{4 \sqrt{r_{2} r_{1}} \cos \left(\frac{\nu_{2}-\nu_{1}}{2}\right)}-\frac{1}{2}, \quad m=\frac{\mu \tau^{2}}{\left[2 \sqrt{r_{2} r_{1}} \cos \left(\frac{\nu_{2}-\nu_{1}}{2}\right)\right]^{3}}, \\
& x=\frac{1}{2}\left[1-\cos \left(\frac{E_{2}-E_{1}}{2}\right)\right], \quad X=\frac{E_{2}-E_{1}-\sin \left(E_{2}-E_{1}\right)}{\sin ^{3}\left(\frac{E_{2}-E_{1}}{2}\right)},
\end{aligned}
$$

$\nu_{2}-\nu_{1}$ is the difference between true anomalies, directly obtained from the observations and $E_{2}-E_{1}$ is the difference between eccentric anomalies, which will be calculated in the process.

The original Gauss' scheme has a restriction when the angle formed by the two position vectors is greater than $\pi / 4$, since in this case the areas of the triangle and the ellipse sector are not similar. For this reason, Danchick in [16] modified Gauss' method solving separately by means of Newton's method the Gaussian equations, depending on the value of the true anomalies. As a consequence, he got to determinate preliminary orbits with a range of the difference of true anomalies until $\pi$ radians and also removed the two other limitation of the classical method: slow convergence and sensitivity to initial guess. In order to improve Danchick's method regarding order of convergence, number of iterations and exact error, we will replace in this procedure Newton's scheme by other high-order methods. Then, we hold the advantages of Danchick's reformulation in terms of initial estimations and range of the true anomalies, improving the velocity of convergence and the precision of the results.

The procedures that we will use to compare the original and the proposed schemes, whose results can be seen in the following tables, are:

- FPoint is the classical Fixed Point method that was originally used by Gauss (see [18]).

- The original method used by Roy Danchick ([16]),

$$
x_{k+1}=x_{k}-\frac{f\left(x_{k}\right)}{f^{\prime}\left(x_{k}\right)} .
$$

- DS is the classical Steffensen's method (see [19]). In it, the derivative of Newton's scheme is replaced by a divided difference of first order $f\left[z_{k}, x_{k}\right]$ with $z_{k}=x_{k}+f\left(x_{k}\right)$,

$$
x_{k+1}=x_{k}-\frac{f^{2}\left(x_{k}\right)}{f\left(z_{k}\right)-f\left(x_{k}\right)} .
$$


- DSR is the derivative-free method obtained by Steffensen's scheme using $z_{k}=x_{k}-f\left(x_{k}\right)$,

$$
x_{k+1}=x_{k}-\frac{f^{2}\left(x_{k}\right)}{f\left(x_{k}\right)-f\left(z_{k}\right)} .
$$

- DT is the classical Traub's method

$$
\begin{array}{r}
y_{k}=x_{k}-\frac{f\left(x_{k}\right)}{f^{\prime}\left(x_{k}\right)}, \\
x_{k+1}=y_{k}-\frac{f\left(y_{k}\right)}{f^{\prime}\left(x_{k}\right)} .
\end{array}
$$

- DTS is the derivative-free version of Traub's method (it can be easily proved that it has third-order of convergence)

$$
\begin{aligned}
y_{k} & =x_{k}-\frac{f^{2}\left(x_{k}\right)}{f\left(z_{k}\right)-f\left(x_{k}\right)}, \quad z_{k}=x_{k}+f\left(x_{k}\right) \\
x_{k+1} & =y_{k}-\frac{f\left(x_{k}\right) f\left(y_{k}\right)}{f\left(z_{k}\right)-f\left(x_{k}\right)} .
\end{aligned}
$$

- The same idea, but using $z_{k}=x_{k}-f\left(x_{k}\right)$, yields DTSR

$$
\begin{aligned}
y_{k} & =x_{k}-\frac{f^{2}\left(x_{k}\right)}{f\left(z_{k}\right)-f\left(x_{k}\right)}, \quad z_{k}=x_{k}-f\left(x_{k}\right) \\
x_{k+1} & =y_{k}-\frac{f\left(x_{k}\right) f\left(y_{k}\right)}{f\left(x_{k}\right)-f\left(z_{k}\right)} .
\end{aligned}
$$

- MO is one of the methods of our new family of Steffensen-type schemes (1), where the used weight functions are:

$$
\begin{aligned}
H(\mu) & =1+\mu, \\
G(\eta) & =1 / 4+(-3 / 4+\eta)^{2}-2(-1+\eta)^{3}+\eta .
\end{aligned}
$$

In Tables 1 to 5 we will see other pieces of information that will help us to compare the methods, as the number of iterations iter and the Approximated Computational Order of Convergence $\rho$ given by (see [20]):

$$
p \approx \rho=\frac{\ln \left(\left|x_{k+1}-x_{k}\right| /\left|x_{k}-x_{k-1}\right|\right)}{\ln \left(\left|x_{k}-x_{k-1}\right| /\left|x_{k-1}-x_{k-2}\right|\right)} .
$$

From $\rho$ we obtain what we call the approximated index of efficiency denoted by $\tilde{I}=\rho^{1 / d}$ and we also use the approximated computational index calculated as $\tilde{I}_{c}=\rho^{1 / o p}$, where $d$ is the number of functional evaluations and op is number of products and quotients realized per step. Finally, error parameter is the exact error of each orbital element.

These numerical results have been obtained with Wolfram Mathematica software, using Variable Precision Arithmetic with 1000 digits and the stopping criterium $\left|x_{k+1}-x_{k}\right|<10^{-100}$. To check these methods, we will calculate the reference orbits that appear in [18]. As a common piece of information of these orbits, we have the next parameters: $k=0.0743654$ (e.r. $)^{\frac{3}{2}} / \min , \mu=1$ (e.m.) and $a_{e}=1$ (e.r.), where $a_{e}$ is the distance between the mass center and the focus of the ellipse where the Earth is situated; e.r. is a unit of distance relative to the equatorial radius of the Earth, $1 e . r \approx 6373.0024 \mathrm{~km}$ and e.m. is a unit relative to the center of masses of the Earth. Also we must comment that J.D. is equal to Julian Days and $\mathrm{T}$ is the time of pass through the perigee.

\section{- Reference Orbit I}

Position vectors

$$
\begin{aligned}
& \vec{r}_{1} \approx[2.460809287053385,2.040522906364322,0.143819057688152], \\
& \vec{r}_{2} \approx[1.988041555748197,2.503333545052242,0.314553506052512] .
\end{aligned}
$$




\section{Orbital parameters}

\begin{tabular}{c|c|c}
\hline$a=4.0$ e.r. & $i=15^{\circ}$ & $\Delta t=0.01044412$ J.D. \\
$e=0.2$ & $\Omega=30^{\circ}$ & \\
$T$ = January $1,19640^{h r}, 0^{\text {min }}, 0^{\text {seg }}$ & $\omega=10^{\circ}$ & \\
\hline
\end{tabular}

\begin{tabular}{||l|c|c|c|c|c|c|c|c||}
\hline \hline & FPoint & Danchick & DS & DSR & DT & DTS & DTSR & MO \\
\hline iter & 53 & 5 & 5 & 5 & 4 & 4 & 3 & 3 \\
\hline$\rho$ & 0.9999 & 2.0000 & 2.0000 & 1.9999 & 3.0000 & 3.0000 & 2.9999 & 8.0010 \\
\hline$\tilde{I}$ & 0.9987 & 1.3945 & 1.3812 & 1.4673 & 1.6969 & 1.4531 & 1.5424 & 1.7313 \\
\hline$\tilde{I}_{c}$ & 0.9974 & 1.9459 & 1.3812 & 1.4673 & 2.2420 & 1.4531 & 1.5424 & 1.1575 \\
\hline error $_{a}$ & $3.6805 \mathrm{e}-104$ & $5.7675 \mathrm{e}-125$ & $1.7318 \mathrm{e}-115$ & $1.0414 \mathrm{e}-183$ & $1.5440 \mathrm{e}-145$ & $4.8431 \mathrm{e}-200$ & $4.3307 \mathrm{e}-130$ & $4.8431 \mathrm{e}-200$ \\
\hline error $_{e}$ & $5.7528 \mathrm{e}-104$ & $6.6345 \mathrm{e}-125$ & $1.9922 \mathrm{e}-115$ & $1.1980 \mathrm{e}-183$ & $1.7761 \mathrm{e}-145$ & $8.8035 \mathrm{e}-201$ & $4.9817 \mathrm{e}-130$ & $8.8035 \mathrm{e}-201$ \\
\hline error $_{i}$ & $2.2349 \mathrm{e}-102$ & $2.4417 \mathrm{e}-123$ & $7.3317 \mathrm{e}-114$ & $4.4089 \mathrm{e}-182$ & $6.5365 \mathrm{e}-144$ & $3.9324 \mathrm{e}-200$ & $1.8334 \mathrm{e}-128$ & $3.9324 \mathrm{e}-200$ \\
\hline error $_{\omega}$ & $7.9614 \mathrm{e}-102$ & $8.6981 \mathrm{e}-123$ & $2.6118 \mathrm{e}-113$ & $1.5706 \mathrm{e}-181$ & $2.3285 \mathrm{e}-143$ & $1.4009 \mathrm{e}-199$ & $6.5313 \mathrm{e}-128$ & $1.4009 \mathrm{e}-199$ \\
\hline error $_{\Omega}$ & 0 & 0 & 0 & 0 & 0 & 0 & 0 & 0 \\
\hline Time & 0.0038418 & 0.0594093 & 0.1227397 & 0.1313144 & 0.0522505 & 0.1112143 & 0.0821334 & 0.0029408 \\
\hline \hline
\end{tabular}

Table 1: Results of Reference Orbit I, $\left|\nu_{2}-\nu_{1}\right|=12.2319^{\circ}$, initial estimation $y_{0}=1$

In the first orbit, the difference of true anomalies is about 12 degrees, thus the initial approximation can be $y_{0}=1$ since this value of true anomalies is below forty-five degrees (this is, the areas of the sector-triangle are similar). For this reason, Fixed Point gives us the solution but needs a high number of iterations. DTSR and MO reach the solution in only three iterations but $\mathrm{MO}$ is more accurate, using the least computational time.

- Reference Orbit II

Position vectors

$$
\begin{aligned}
& \vec{r}_{1} \approx[0.411362066797608,-1.662499999999999,0.822724133595216], \\
& \vec{r}_{2} \approx[0.977567529772089,-1.644280060976665,-0.04236299091611] .
\end{aligned}
$$

\section{Orbital parameters}

\begin{tabular}{c|c|c}
\hline$a=2.0$ e.r. & $i=60^{\circ}$ & $\Delta t=0.01316924$ J.D. \\
$e=0.05$ & $\Omega=120^{\circ}$ & \\
$T$ = December 23, $19630^{h r}, 0^{\text {min }}, 0^{\text {seg }}$ & $\omega=150^{\circ}$ & \\
\hline
\end{tabular}

Looking at Table 2, the difference of true anomalies is greater than in the previous one, but we can use the same initial approximation since the difference of true anomalies is below forty-five degrees. Now, the number of iterations in Fixed Point is the highest, that is, the originally Gauss' method loses performance with increasing the difference of true anomalies. As we can see DT, DTS, DTSR and MO reach the orbital parameters with the same error but MO needs only three iterations and continue having the least computational time.

\section{- Reference Orbit III} Position vectors

$$
\begin{aligned}
& \vec{r}_{1} \approx[-2.578226630220951,2.136491364121168,0.590044146022302], \\
& \vec{r}_{2} \approx[3.4985306433286335,-2.946150606082265,0.231098880639301] .
\end{aligned}
$$




\begin{tabular}{||l|c|c|c|c|c|c|c|c||}
\hline \hline & FPoint & Danchick & DS & DSR & DT & DTS & DTSR & MO \\
\hline iter & 100 & 6 & 6 & 6 & 5 & 4 & 4 & 3 \\
\hline$\rho$ & 0.99999 & 2.0000 & 2.0000 & 1.9999 & 3.0000 & 3.0000 & 2.9999 & 8.0126 \\
\hline$\tilde{I}$ & 0.9990 & 1.3876 & 1.3667 & 1.4419 & 1.4886 & 1.4215 & 1.4945 & 1.7179 \\
\hline$\tilde{I}_{c}$ & 0.9980 & 1.9289 & 1.3667 & 1.4419 & 1.8174 & 1.4215 & 1.4945 & 1.1552 \\
\hline error $_{a}$ & $7.6194 \mathrm{e}-103$ & $3.6639 \mathrm{e}-137$ & $8.4958 \mathrm{e}-116$ & $2.4566 \mathrm{e}-200$ & $7.1500 \mathrm{e}-202$ & $7.1508 \mathrm{e}-202$ & $7.1508 \mathrm{e}-202$ & $7.1508 \mathrm{e}-202$ \\
\hline error $_{e}$ & $6.6292 \mathrm{e}-102$ & $2.2202 \mathrm{e}-136$ & $5.1481 \mathrm{e}-115$ & $1.4425 \mathrm{e}-199$ & $2.7300 \mathrm{e}-202$ & $2.7300 \mathrm{e}-202$ & $2.7325 \mathrm{e}-202$ & $2.7325 \mathrm{e}-202$ \\
\hline error $_{i}$ & $1.0392 \mathrm{e}-101$ & $3.3928 \mathrm{e}-136$ & $7.8671 \mathrm{e}-115$ & $2.1984 \mathrm{e}-199$ & $1.0162 \mathrm{e}-201$ & $1.0162 \mathrm{e}-201$ & $1.0163 \mathrm{e}-201$ & $1.0163 \mathrm{e}-201$ \\
\hline error $_{\omega}$ & $9.6868 \mathrm{e}-100$ & $3.1625 \mathrm{e}-134$ & $7.3332 \mathrm{e}-113$ & $2.0492 \mathrm{e}-197$ & $9.4730 \mathrm{e}-200$ & $9.4730 \mathrm{e}-200$ & $9.4732 \mathrm{e}-200$ & $9.4732 \mathrm{e}-200$ \\
\hline error $_{\Omega}$ & 0 & 0 & 0 & 0 & 0 & 0 & 0 & 0 \\
\hline Time & 0.0050993 & 0.0698852 & 0.1527216 & 0.1644351 & 0.0675880 & 0.1066893 & 0.1141550 & 0.0030695 \\
\hline \hline
\end{tabular}

Table 2: Results of Reference Orbit II, $\left|\nu_{2}-\nu_{1}\right|=31.4649^{\circ}$, initial estimation $y_{0}=1$

\section{Orbital parameters}

\begin{tabular}{c|c|c}
\hline$a=4.0$ e.r. & $i=88^{\circ}$ & $\Delta t=0.21227310$ J.D. \\
$e=0.15$ & $\Omega=140^{\circ}$ & \\
$T$ = January 30, $19640^{h r}, 0^{\text {min }}, 0^{\text {seg }}$ & $\omega=10^{\circ}$ & \\
\hline
\end{tabular}

\begin{tabular}{||l|c|c|c|c|c|c|c|c||}
\hline \hline & FPoint & Danchick & DS & DSR & DT & DTS & DTSR & MO \\
\hline iter & - & - & - & - & - & - & - & 4 \\
\hline$\rho$ & - & - & - & - & - & - & - & 8.0235 \\
\hline$\tilde{I}$ & - & - & - & - & - & - & - & 1.5210 \\
\hline$\tilde{I}_{c}$ & - & - & - & - & - & - & - & 1.1133 \\
\hline error $_{a}$ & - & - & - & - & - & - & - & $1.7358 \mathrm{e}-202$ \\
\hline error $_{e}$ & - & - & - & - & - & - & - & $3.6984 \mathrm{e}-202$ \\
\hline error $_{i}$ & - & - & - & - & - & - & - & $1.2487 \mathrm{e}-202$ \\
\hline error $_{\omega}$ & - & - & - & - & - & - & - & $8.0140 \mathrm{e}-200$ \\
\hline error $_{\Omega}$ & - & - & - & - & - & - & - & $2.4857 \mathrm{e}-251$ \\
\hline Time $^{2}$ & - & - & - & - & - & - & - & 0.00114858 \\
\hline \hline
\end{tabular}

Table 3: Results of Reference Orbit III, $\left|\nu_{2}-\nu_{1}\right|=59.0148^{\circ}$, initial estimation $y_{0}=0.4$

To determine this orbit is more complicate than the previous cases, since the difference of true anomalies is above forty-five degrees, for this reason fixed point scheme can not reach the solution. In Table 3 , we can see that MO method is the only one able to give the solution in spite of we are using a very good initial estimation. Because of this problem of convergence, we decided to represent the dynamical planes of these derivative-free methods to conclude if our methods reach the solution and which points are good initial estimations. From Figures $2 \mathrm{a}$ to $3 \mathrm{~b}$, we deduce that $y_{0}=0.46$ is a good initial approximation for all the methods, since this value is in the basin of attraction of all them. These dynamical planes show the wideness of the regions of good starting points for a particular bound of the error. If this bound is modified, these areas of convergence change in a proportional way.

In Table 4 we can see that, in spite of using the good initial guess $y_{0}=0.46$, the only method able to give us a solution (with the same tolerance as in Orbits I and II) is MO, giving the solution with a small error, being the number of iterations and the computational time specially low. However, by the information obtained from the dynamical planes, all the methods should converge with this initial estimation. Then, we decided to relax the requirements on the methods to ensure that they converge, that is, to use a higher tolerance $t o l=10^{-10}$ and Variable Precision Arithmetics with 250 digits. Under these conditions we can compare all the methods, as we can see in Table 5. 


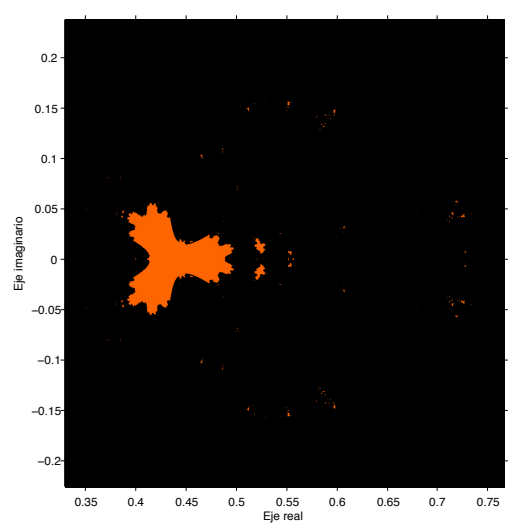

(a) $\mathrm{MO}$

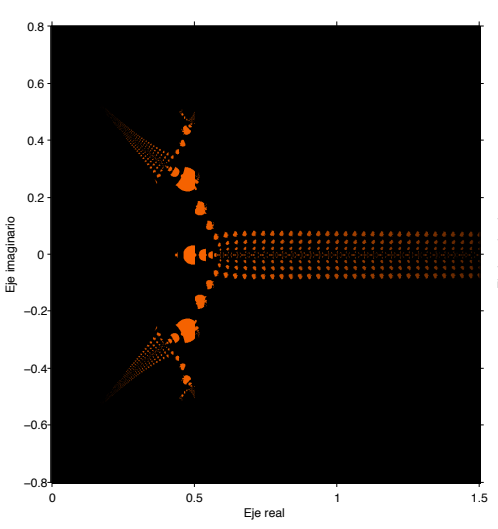

(b) DS

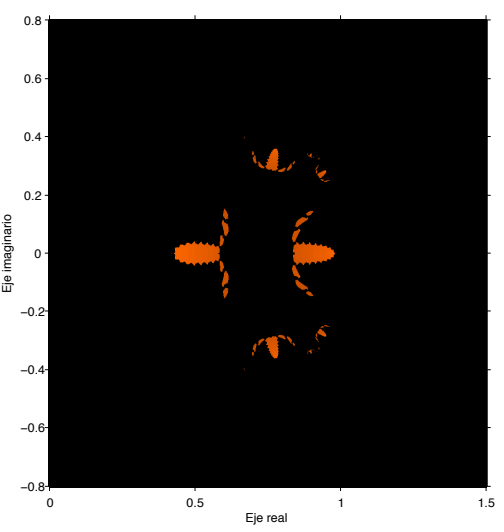

(c) DSR

Figure 2: Dynamical planes of MO, DS and DSR respectively

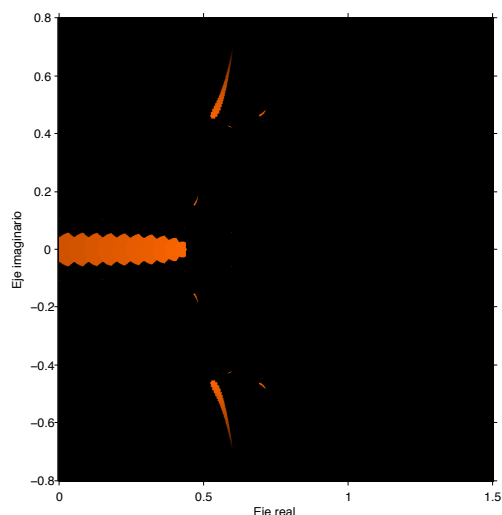

(a) DTS

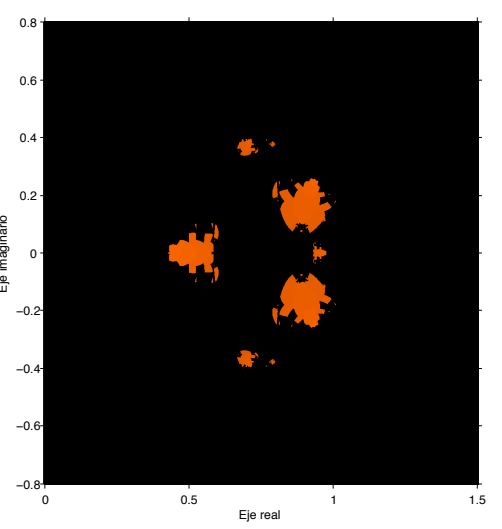

(b) DTSR

Figure 3: Dynamical planes of DTS and DTSR respectively

\begin{tabular}{||l|c|c|c|c|c|c|c|c||}
\hline \hline & FPoint & Danchick & DS & DSR & DT & DTS & DTSR & MO \\
\hline iter & - & - & - & - & - & - & - & 4 \\
\hline$\rho$ & - & - & - & - & - & - & - & 8.0010 \\
\hline$\tilde{I}$ & - & - & - & - & - & - & - & 1.5528 \\
\hline$\tilde{I}_{c}$ & - & - & - & - & - & - & - & 1.1221 \\
\hline error $_{a}$ & - & - & - & - & - & - & - & $1.7358 \mathrm{e}-202$ \\
\hline error $_{e}$ & - & - & - & - & - & - & - & $3.6984 \mathrm{e}-202$ \\
\hline error $_{i}$ & - & - & - & - & - & - & - & $1.2487 \mathrm{e}-202$ \\
\hline error $_{\omega}$ & - & - & - & - & - & - & - & $8.0140 \mathrm{e}-200$ \\
\hline error $_{\Omega}$ & - & - & - & - & - & - & - & $2.4857 \mathrm{e}-251$ \\
\hline Time & - & - & - & - & - & - & - & 0.00121789 \\
\hline \hline
\end{tabular}

Table 4: Results of Reference Orbit III, tol $=10^{-100},\left|\nu_{2}-\nu_{1}\right|=59.0148^{\circ}$, initial estimation $y_{0}=0.46$

DT and DTS need only two iterations to give us the solutions. However, if we look at the error of the orbital parameters we can see that MO gives us the least error in the shortest computational time, with only one more iteration. 


\begin{tabular}{||l|c|c|c|c|c|c|c|c||}
\hline \hline & FPoint & Danchick & DS & DSR & DT & DTS & DTSR & MO \\
\hline iter & - & 3 & 7 & 5 & 2 & 2 & 4 & 3 \\
\hline$\rho$ & - & 1.9964 & 2.0068 & 2.0182 & 2.0974 & 2.0974 & 2.9721 & 8.0010 \\
\hline$\tilde{I}$ & - & 1.2536 & 1.1929 & 1.2801 & 0.9136 & 1.2251 & 1.4884 & 1.5528 \\
\hline$\tilde{I}_{c}$ & - & 1.5969 & 1.1929 & 1.1977 & 1.4482 & 0.9136 & 1.2251 & 1.1088 \\
\hline error $_{a}$ & - & $5.5124 \mathrm{e}-10$ & $1.7154 \mathrm{e}-12$ & $1.1879 \mathrm{e}-09$ & $3.2784 \mathrm{e}-10$ & $3.2784 \mathrm{e}-10$ & $1.3312 \mathrm{e}-14$ & $1.7359 \mathrm{e}-202$ \\
\hline error $_{e}$ & - & $1.7082 \mathrm{e}-10$ & $5.3064 \mathrm{e}-13$ & $3.6812 \mathrm{e}-10$ & $1.0160 \mathrm{e}-10$ & $1.0160 \mathrm{e}-10$ & $2.2672 \mathrm{e}-15$ & $3.6985 \mathrm{e}-202$ \\
\hline error $_{i}$ & - & $1.6482 \mathrm{e}-11$ & $5.1001 \mathrm{e}-14$ & $3.5518 \mathrm{e}-11$ & $9.8032 \mathrm{e}-12$ & $9.8032 \mathrm{e}-12$ & $1.7243 \mathrm{e}-16$ & $1.2488 \mathrm{e}-202$ \\
\hline error $_{\omega}$ & - & $1.0577 \mathrm{e}-08$ & $3.2730 \mathrm{e}-11$ & $2.2793 \mathrm{e}-08$ & $6.2911 \mathrm{e}-09$ & $6.2911 \mathrm{e}-09$ & $1.1065 \mathrm{e}-13$ & $8.0140 \mathrm{e}-200$ \\
\hline error $_{\Omega}$ & - & $2.4800 \mathrm{e}-251$ & $2.4800 \mathrm{e}-251$ & $2.4800 \mathrm{e}-251$ & $2.4800 \mathrm{e}-251$ & $2.4800 \mathrm{e}-251$ & $2.4800 \mathrm{e}-251$ & $2.4800 \mathrm{e}-251$ \\
\hline Time $^{-}$ & - & 0.002872 & 0.018754 & 0.010852 & 0.001832 & 0.014793 & 0.007634 & 0.000931 \\
\hline \hline
\end{tabular}

Table 5: Results of Reference Orbit III, $t o l=10^{-10},\left|\nu_{2}-\nu_{1}\right|=59.0148^{\circ}$, initial estimation $y_{0}=0.46$

\section{Conclusions}

In this paper, we have designed a family of Steffensen-type methods of optimal eighth-order by using weight functions procedure. We have also used the Danchick-Gauss' method for preliminary orbit determination and we have improved it by replacing Newton's method by the proposed ones, holding both the amplitude of the range of the true anomalies and the sensitivity to the initial guess. Indeed, we reduce considerably the number of iterations and the error in the calculations. Moreover, from the dynamical planes it can be inferred that the modified eighth-order method is much more stable than the original from Danchick which used Newton's scheme.

\section{References}

[1] D.D. Bruns, J.E. Bailey, Nonlinear feedback control for operating a nonisothermal CSTR near an unstable steady state, Chemical Engineering Science 32 (1977) 257-264.

[2] J.A. Ezquerro, J.M. Gutiérrez, M.A. Hernández, M.A. Salanova, Chebyshev-like methods and quadratic equations, Revue d'Analyse Numérique et de Théorie de l'Approximation 28 (1999) 23-35.

[3] Y. Zhang, P. Huang, High-precision Time-interval Measurement Techniques and Methods, Progress in Astronomy 24(1) (2006) 1-15.

[4] Y. He, C. Ding, Using accurate arithmetics to improve numerical reproducibility and stability in parallel applications, Journal of Supercomputing 18 (2001) 259-277.

[5] A.M. Ostrowski, Solutions of equations and systems of equations, Academic Press, New York-London, 1966.

[6] H. T. Kung, J. F. Traub, Optimal order of one-point and multi-pointiteration, Journal Assoc. Comput. Math. 21 (1974) 643-651.

[7] M.S. Petković, B. Neta, L.D. Petković, J. Džunić, Multipoint methods for solving nonlinear equations, Elsevier, 2013.

[8] J.M. Ortega, W.G. Rheinboldt, Iterative solutions of nonlinear equations in several variables, Academic Press, New York, 1970.

[9] A. Cordero, J. R. Torregrosa, A class of Steffensen type methods with optimal order of convergence, Applied Mathematics and Computation 217 (2011) 7653-7659.

[10] Q. Zheng, J. Li, F. Huang, An optimal Steffensen-type family for solving nonlinear equations, Applied Mathematics and Computation 217 (2011) 9592-9597. 
[11] P. Sargolzaei, F. Soleymany, Accuracy eighth- and fourteenth-order methods for solving nonlinear equations, Numerical Algorithms 58 (2011) 513-527.

[12] A. Cordero, J.L. Hueso, E. Martínez, J.R. Torregrosa, A new technique to obtain derivative-free optimal iterative methods for solving nonlinear equations, Journal of Computational and Applied Mathematics, 252 (2013) 95-102.

[13] F. Soleymany, S. Shateyi, Two optimal eighth-order derivative-free classes of iterative methods, Abstract and Applied Analysis, Vool. 2012, Article ID 318165, 14 pages, doi: 10.1155/2012/318165.

[14] X. Wang, J. Džunić, T. Zhang, On an efficient family of derivative free three-point methods for solving nonlinear equations, Applied Mathematics and Computation 219 (2012) 1749-1760.

[15] R. Thukral, New Sixteenth-Order Derivative-Free Methods for Solving Nonlinear Equations, American Journal of Computational and Applied Mathematics 2(3) (2012) 112-118.

[16] R. Danchick, Gauss meets Newton again: How to make Gauss orbit determination from two position vectors more efficient and robust with Newton-Raphson iterations, Applied Mathematics and Computation 195 (2008) 364-375.

[17] A. Cordero, J. R. Torregrosa, A technique to design derivative-free methods for nonlinear equations, Proceedings of the 13th International Conference on Computational and Mathematical Methods in Science and Engineering, CMMSE 2013, 24-27 June, 2013, ISBN: 978-84-616-2723-3.

[18] Pedro Ramon Escobal, Methods of Orbit Determination, Krieger Publishing Company, 1975.

[19] J.F. Traub, Iterative methods for the solution of equations,New York, Chelsea Publishing Company, 1982.

[20] A. Cordero, J.R. Torregrosa. Variants of Newton's Method using fifth-order quadrature formulas. Applied Mathematics and Computation 190 (2007) 686-698. 